\title{
3-P-126 Poster Sessions
}

\section{Possible involvement of peroxynitrite in promotion of proliferative activity in the neural stem/progenitor cells after hippocampal dentate granule cell loss}

\author{
Masanori Yoneyama ${ }^{1}$, Yoshimi Ikeda ${ }^{1}$, Taro Yamaguchi', Yusuke Onaka ${ }^{1}$, Kiyokazu Ogita ${ }^{1}$
}

${ }^{1}$ Laboratory of Pharmacology, Faculty of Pharmaceutical Sciences, Setsunan University

Our previous studies demonstrated that the trimethyltin chloride (TMT) causes the granule cell loss in the dentate gyrus (DG) of adult mouse, with being regenerated in the dentate granule cell after the neuronal loss. To elucidate the involvement of peroxynitrite in proliferation of neural stem/progenitor cells (NPCs) after neuronal degeneration, we evaluated the expression of 3-nitrotyrosine (3-NT, a product of tyrosine nitration by peroxynitrite) in the newly generated cells following neurodegeneration in the DG. Mice were given TMT to prepare slices for immunostaining using antibody against nestin (NPCs marker) and 3-NT. Cells positive for nestin and 3-NT markedly increased in the DG on day 3 after TMT treatment. In vitro experiments using the NPCs isolated from the DG on day 3 post-TMT, exposure to apocynin (NADPH oxidase inhibitor) or L-NAME (nitric oxide synthase inhibitor) significantly attenuated the cell proliferation. However, KT5823 (G kinase inhibitor) did not affect it. These results support the possibility that peroxynitrite promotes proliferative activity of the NPCs generated following neuronal degeneration in the DG. 